\title{
Study on Rail Profile Optimization Based on the Nonlinear Relationship between Profile and Wear Rate
}

\author{
Jianxi Wang, ${ }^{1,2}$ Zhiqiang Ren, ${ }^{1,3}$ Jinjie Chen, ${ }^{4}$ and Long Chen ${ }^{4}$ \\ ${ }^{1}$ School of Civil Engineering, Shijiazhuang Tiedao University, Shijiazhuang 050043, China \\ ${ }^{2}$ Key Laboratory of Road and Railway Engineering Safety Control of Ministry of Education, Shijiazhuang Tiedao University, \\ Shijiazhuang 050043, China \\ ${ }^{3}$ China Railway Fifth Survey and Design Institute Group Co., Ltd., Beijing 102600, China \\ ${ }^{4}$ School of Transportation Engineering, Shijiazhuang Tiedao University, Shijiazhuang 050043, China
}

Correspondence should be addressed to Jianxi Wang; qianxi-2008@163.com

Received 3 November 2016; Revised 3 May 2017; Accepted 22 May 2017; Published 20 June 2017

Academic Editor: George S. Dulikravich

Copyright (C) 2017 Jianxi Wang et al. This is an open access article distributed under the Creative Commons Attribution License, which permits unrestricted use, distribution, and reproduction in any medium, provided the original work is properly cited.

\begin{abstract}
This paper proposes a rail profile optimization method that takes account of wear rate within design cycle so as to minimize rail wear at the curve in heavy haul railway and extend the service life of rail. Taking rail wear rate as the object function, the vertical coordinate of rail profile at range optimization as independent variable, and the geometric characteristics and grinding depth of rail profile as constraint conditions, the support vector machine regression theory was used to fit the nonlinear relationship between rail profile and its wear rate. Then, the profile optimization model was built. Based on the optimization principle of genetic algorithm, the profile optimization model was solved to achieve the optimal rail profile. A multibody dynamics model was used to check the dynamic performance of carriage running on optimal rail profile. The result showed that the average relative error of support vector machine regression model remained less than $10 \%$ after a number of training processes. The dynamic performance of carriage running on optimized rail profile met the requirements on safety index and stability. The wear rate of optimized profile was lower than that of standard profile by $5.8 \%$; the allowable carrying gross weight increased by $12.7 \%$.
\end{abstract}

\section{Introduction}

Increasing the travel speed of carriage and the axle load constitutes the two key development orientations of heavy haul railway, which are considered the primary cause of rail wear aggravation. The increase in rail wear rate results in the reduction of renewal rail interval and the increase in the transport cost on heavy haul railway, especially at its curved sections. According to statistics, curved sections account for about one-third of total mileage of railways in China [1], and lots of small-radius curved sections are untimely changed due to serious rail side wear, which causes the increase in maintenance costs and impairs train working safety. With rail profile as its object of study, this paper proposes rationally optimized design of rail profile so as to minimize rail wear loss during the travel of carriage, thus being of important engineering significance for heavy haul railway transport.
Scholars at home and abroad extensively studied rail profile optimization design. Rolling circle radius difference, as one of the key parameters describing the contact between wheelset and rail, determines dynamic characteristics and affects the curve negotiating performance of train. Many scholars optimized wheel-rail profile by using rolling radius difference as object function. Magel and Kalousek (2002) [2] proposed the profile optimization design scheme based on the rolling circle radius difference between left and right wheels through wheel-rail contact mechanics analysis. Shevtsov (2008) [3] analyzed the relationship between rail profile and wear index and fatigue index, mitigate rolling contact fatigue by shifting the contact point from rolling contact fatigue area to the rail top, and defined the criteria for selection of wheelset rolling circle radius difference (RRD) as "no wheel-rail contact point in rolling contact fatigue area." Appropriate RRD curves were selected in this way for 
optimization of rail profile. Jia and Si (2014) [4] and Zhai et al. (2014) [5] determined the rational wheel-diameter difference and improved the curve negotiating performance through the analysis of rail wear characteristics at small-radius curved sections of the Shuo-Huang Railway, and a rail grinding target profile was designed to reduce wheel-rail contact stress and mitigate rolling contact fatigue.

High rail of small-radius curved section suffers from serious wear and fatigue damage. Many scholars carried out rail profile optimization directly with wear or fatigue damage as object function. In consideration of the large number of curved sections along light rail and the high wheel-rail maintenance costs, Brandau et al. (2002) [6] performed asymmetric profile optimization design of rail head at curved section and changed the rail profile of specific curved section, having thereby greatly reduced wheel-rail wear loss without impairing the operation of carriages at other sections. Choi et al. (2013) [7] proposed an asymmetric profile optimization design method with rail wear index as object function and wheel-rail dynamic response characteristics as the constraint condition, which helped to minimize the serious rail wear at curved sections of urban rail transit. Xiao (2011) [8] proposed a multiobjective rail profile optimization scheme designed principally to reduce fatigue and wear at curved section based on the relationship between wheel-rail contact mechanics and wheel-rail geometry. Dollevoet (2010) [9] believed that asymmetric rail profile should be the most effective way to eliminate rail contact fatigue. He discovered by dint of nonHertz contact theory that the shear stress of rail shoulder where rail contact fatigue crack appears was relatively high. He designed a new profile based on rail contact fatigue crack initiation mechanism so as to minimize the shearing stress that was produced by rail shoulder out of wheel-rail contact, thereby alleviating rolling contact fatigue crack of rail. Cui et al. (2011) [10] performed profile optimization design so as to improve the conformal degree of wheel-rail contact and reduce the normal clearance of wheel-rail contact and sought numerical solution of mathematical model for profile optimization through sequential quadratic programming. Persson et al. (2010) [11] optimized the profile of curved rail through genetic algorithm based on wheel-rail dynamics and numerical simulation theory. Switch plays an important role in railway operation. The change in rail profile at switch area brings about intense wheel-rail dynamic response, poor safety, and frequent rail wear and damage. Pålsson (2013) [12] proposed the rail profile solution seeking based on NSGA-II genetic algorithm with contact stress and contact spot energy dissipation as object function so as to minimize dynamic response and rail wear and damage at switch area.

Since wheel-rail contact geometry has an immediate impact on wheel-rail damage, the optimization of wheelrail contact relationship could effectively alleviate wheel-rail damage. Shen and Zhong (2014) [13] proposed a contact angle curve inverse estimation method for wheel-rail profile design. This method determines the relationship between profile contour and contact angle value through integral, reversely obtains target profile by correcting the contact angle curve, and brings about a new wheel-rail profile through repeated correction. Jahed et al. (2008) [14] performed optimization design of wheel profile using mathematical method with wheel-rail contact parameter as object variable. Gerlici and Lack (2011) [15] optimized wheel and rail profiles through arc radius profile change-based repeated iteration based on the analysis of expected shape of wheel/rail contact geometry. Wang et al. (2016) [16] established wheel-rail contact point distribution object function using contact geometry theory with the discrete point of rail profile as variable and optimized the design of new rail profile through single-objective nonlinear optimization algorithm so as to minimize rail wear.

Wheel-rail profile optimization is a complex engineering optimization design issue. It is a favorable way to optimize rail profile through the combination of theory and practice. Zhou et al. (2010) [17] studied the preventive grinding of rail, probed into the locations and characteristics of rail damage, and proposed a preventive grinding optimization design scheme for rail based on empirical design method in consideration of the cyclic grinding quantity of rail grinder. Zakharov et al. (2008) [18] summarized the principle of and hands-on experience in wheel-rail selection for Russian railways and introduced a wheel-rail profile evaluation and optimization method established based on rational and practical method. Modern railroad car system is normally designed with embedded track and channel rail. Bergeman (2015) [19] designed asymmetric profile of channel rail based on current rail manufacturing capacity, which has been extensively used for urban rail transit system.

According to above-noted research findings, rail profile is considered invariable and free from the impact of wearinduced change in rail profile during wheel-rail contact in respect of rail profile optimization. The rail optimization result is usually the current simulation comparison result for demonstrating new wheel-rail profile matching effect without considering the fact that rail profile is subjected to wear all the time during operation, that the rail profile changes all the time due to wear, and that the worn wheel-rail profile contact relationship changes therewith. From this point of view, the current wheel-rail contact relationship in optimum state is not in the same position to assure the optimal matching effect after the operation of train for a period of time. Hence, this paper proposes a rail profile optimization method that takes account of wear rate within design cycle. The maximum wear depth, that is, $1 \mathrm{~mm}$, was taken as design cycle. This method updates rail profile through wear loss calculation based on random irregularity on rail with optimized profile as the initial profile, subjects the updated rail profile to another dynamics simulation, calculates the wear loss of updated rail profile, and then works out the wear rate. Since the final profile is immediately associated with initial design profile and the contact geometry state during the operation of train, the paper established the nonlinear relationship between wear rate and rail profile geometric parameter within design cycle through nonlinear fitting, built the optimization model, sought to solve the model with optimization algorithm, and sought an optimized rail profile with the minimum wear rate. The optimization process is shown in Figure 1. 


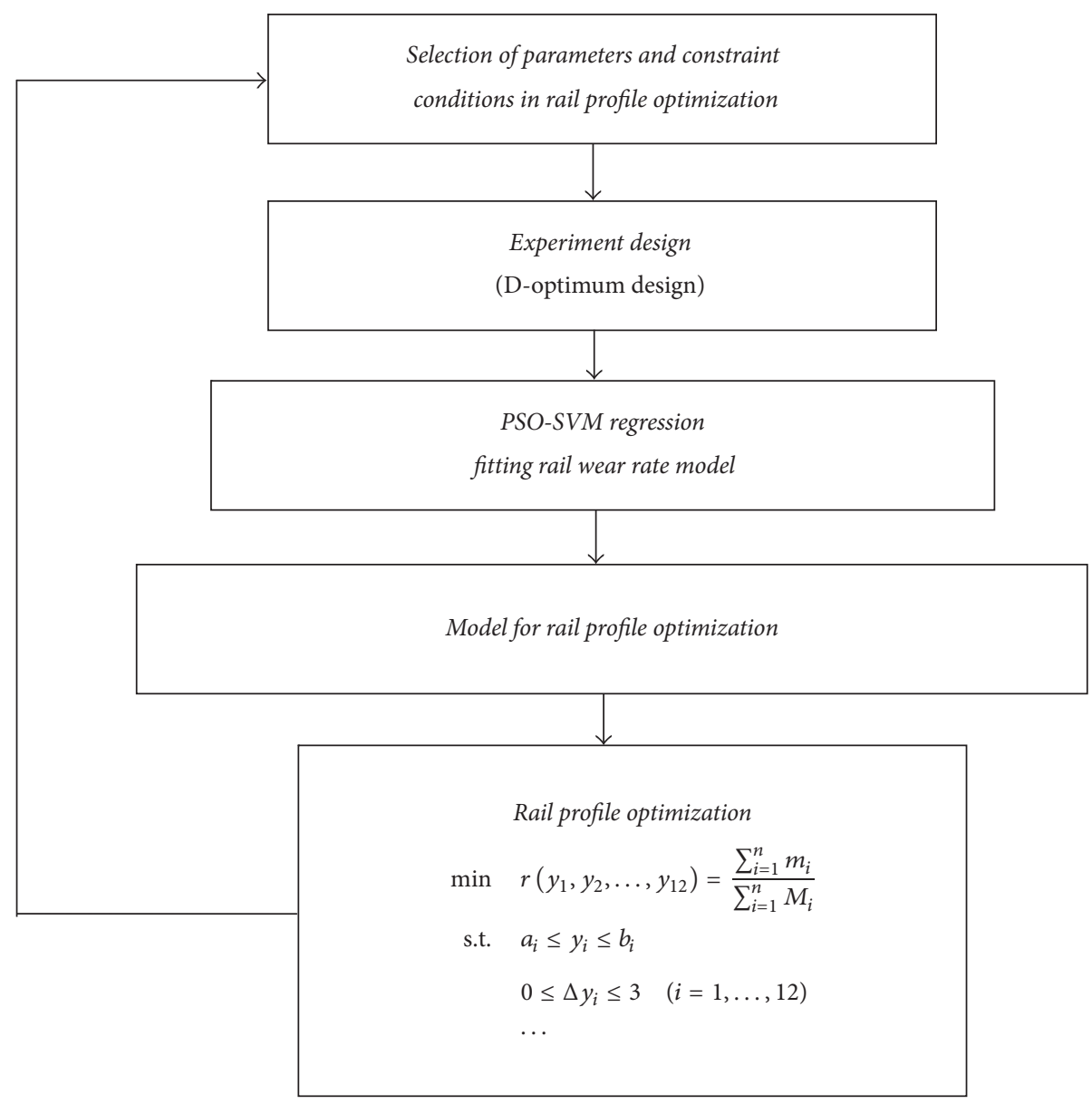

FIGURE 1: Flowchart of rail profile optimal design.

\section{Mathematical Model for Rail Profile Optimization}

This paper studied the regular pattern of rail wear at smallradius curved section of heavy haul railway and established a numerical model for profile optimization with the high rail profile at curve radius of $600 \mathrm{~m}$ along a chosen heavy haul railway. Assuming that the coordinate of rail head crosssectional point is $\left(x_{i}, y_{i}\right)$ and the object function is wear rate $F(x, y)$, then the rail profile could be expressed as spline interpolation function $f(x, y)$ of rail head coordinate point. The mathematical model between rail wear rate and profile is nonlinear mapping relationship $\varphi$; let

$$
\varphi: f(x, y) \longrightarrow F(x, y) .
$$

Subject the mathematical model to optimal solution to get the rail profile with the minimum wear rate.

2.1. Determination of Independent Variable. According to research findings, premature renewal rail is necessary for $\mathrm{CHN} 75 \mathrm{~kg} / \mathrm{m}$ rails in general use for heavy haul railways in China due to side wear when the carrying gross weight reaches approx. $350 \mathrm{Mt}$ (2013) [20]. Figure 2 [4] shows the wear evolution of high rail profile at curved section of a heavy

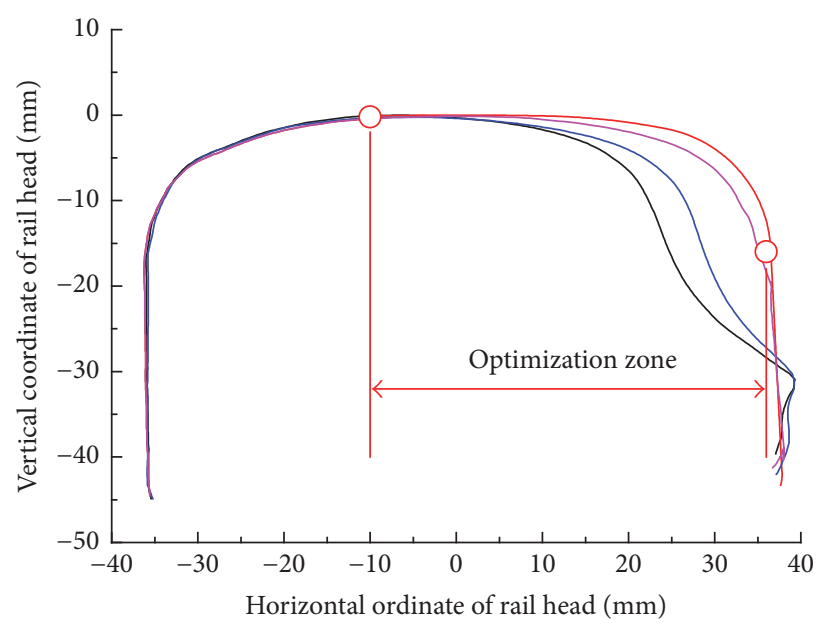

FIgURE 2: Diagram of rail head optimization range (unit: $\mathrm{mm}$ ).

haul railway with curved section radius of $600 \mathrm{~m}$ designed with standard CHN $75 \mathrm{~kg} / \mathrm{m}$ rails.

As shown in Figure 2, the seriously worn points of high rail are principally located between rail center line and 
TABLE 1: Value range of rail profile optimization point (unit: $\mathrm{mm}$ ).

\begin{tabular}{lcccccccccccccc}
\hline $\begin{array}{l}\text { Vertical } \\
\text { coordinate }\end{array}$ & $y_{0}$ & $y_{1}$ & $y_{2}$ & $y_{3}$ & $y_{4}$ & $y_{5}$ & $y_{6}$ & $y_{7}$ & $y_{8}$ & $y_{9}$ & $y_{10}$ & $y_{11}$ & $y_{12}$ & $y_{13}$ \\
$\begin{array}{l}\text { Horizontal } \\
\text { coordinate }\end{array}$ & -10 & 0 & 5 & 10 & 15 & 20 & 23.9 & 26 & 28 & 30 & 32 & 34 & 35 & 36 \\
$\begin{array}{l}\text { Vertical } \\
\text { coordinate } \\
\text { max. value }\end{array}$ & -0.096 & 0.000 & -0.025 & -0.096 & -0.352 & -0.925 & -1.599 & -2.175 & -3.047 & -4.309 & -6.109 & -8.822 & $-10.945-16.000$ \\
$\begin{array}{l}\text { Vertical } \\
\text { coordinate } \\
\text { min. value }\end{array}$ & -0.096 & -3.000 & -3.025 & -3.096 & -3.352 & -3.925 & -4.599 & -5.175 & -6.047 & -7.309 & -9.109 & -11.822 & $-13.945-16.000$ \\
\hline
\end{tabular}

gauge side. Hence, the points within rail profile optimization interval correspond to horizontal coordinate $-10 \sim 36 \mathrm{~mm}$. 12 discrete points are chosen within optimization interval as optimization points. In view of the fact that rail wear principally changes the vertical coordinate of rail profile point, the independent variable in mathematical model for rail profile optimization should be $\mathbf{y}=\left(y_{1}, y_{2}, \ldots, y_{12}\right)^{\mathrm{T}}$.

2.2. Determination of Dependent Variable. Rail wear is a process of accumulation during operation of carriages. The wear loss variation mechanism of different rail profiles is not constant within overall interval for different segments of a time interval. Since the grinding for rail profile optimization is performed within a certain cycle, the rail wear loss shall be changed at determination of object variable so as to avoid local optimum. Expressing the service efficiency of rail as rail wear rate within section is a better way to reflect the wear accumulation process. Take $T$ as the time interval of design cycle, and divide $T$ into $n$ intervals, $m_{i}$ is the rail wear within the $i$ th interval, $M_{i}$ represents the number of carriages passing within the $i$ th interval, and then the wear rate could be expressed as

$$
r=\frac{\sum_{i=1}^{n} m_{i}}{\sum_{i=1}^{n} M_{i}}
$$

Take the unit length of rail at maximum wear depth as the object of study, and the accumulative wear loss would be

$$
\sum_{i=1}^{n} m_{i}=\left[S_{0}\left(y_{1}^{n}, y_{2}^{n}, \ldots, y_{12}^{n}\right)-S_{T}\left(y_{1}^{0}, y_{2}^{0}, \ldots, y_{12}^{0}\right)\right] \cdot l,
$$

where the rail length $l$ is equal to 1 , that is, the unit length. $S_{0}$ and $S_{T}$, respectively, represent the area between initial rail profile and horizontal coordinate axis and the area between rail profile and horizontal coordinate axis after time $T$.

The number of carriages which allowed passing through rail profile within the maximum wear depth could be obtained through dynamics simulation. The object variable is obtained with (2) and (3).

2.3. Determination of Constraint Condition. According to wheel-rail contact relationship and the pertinent literature (2009) [21], the determination of rail profile must meet convex curve condition; that is to say, the slope of segment between consecutive points decreases with the increase of horizontal coordinate. The following constraints shall be imposed for vertical coordinate of the 12 optimization points:

$$
\frac{y_{i}-y_{i-1}}{x_{i}-x_{i-1}}-\frac{y_{i+1}-y_{i-1}}{x_{i+1}-x_{i-1}}>0 \quad(i=1, \ldots, 12) .
$$

Since rail profile is optimized based on original standard profile, the optimized profile is below the original profile, while the optimization of rail profile shall meet the requirements on max. grinding depth (3 $\mathrm{mm}$ in this paper); that is,

$$
\begin{gathered}
a_{i} \leq y_{i} \leq b_{i} \quad(i=1, \ldots, 12), \\
0 \leq \Delta y_{i} \leq 3 \quad(i=1, \ldots, 12),
\end{gathered}
$$

where $a_{i}$ and $b_{i}$ are the lower and upper limits at point $i$.

The curve consisting of optimization points must be smooth and gentle and comply with geometry requirements for rail under practical conditions. To sum up, the coordinates and value range of optimization point in rail profile optimization model are as shown in Table 1.

The mathematical model established for rail profile optimization based on analysis of each element of single-object optimization model is as follows:

$$
\begin{array}{ll}
\min & r\left(y_{1}, y_{2}, \ldots, y_{12}\right)=\frac{\sum_{i=1}^{n} m_{i}}{\sum_{i=1}^{n} M_{i}} \\
\text { s.t. } & a_{i} \leq y_{i} \leq b_{i} \\
& 0 \leq \Delta y_{i} \leq 3 \\
& (i=1, \ldots, 12) \\
& D\left(y_{1}, y_{2}, \ldots, y_{12}\right) \leq[d]
\end{array}
$$

where $D\left(y_{i}\right)$ is the dynamics index function of corresponding rail profile and $[d]$ represents the limit of index corresponding to normal operation of carriage.

\section{PSO-SVM Regression Fitting Rail Wear Rate Model}

There is a complex nonlinear relationship between rail wear rate and rail profile. The dependent variable and independent 
variable are subjected to nonlinear regression through given sample data based on principle of statistics. A mathematical model with generalization capability is attained through sample training for machine learning. Machine learning is designed to handle any complex nonlinear relationship. It converts input and output data by seeking the "black box" of hidden layer, but the complex model obtained through training cannot be expressed with an explicit relation. This paper identifies the nonlinear relationship between rail wear rate and rail profile in this way.

3.1. The Sample of Rail Wear Rate. The sample for data fitting (the wear rate of different rail profiles) can be obtained by field measurements and multibody dynamics simulations. Due to the number of rail wear observations, they are unable to meet the sampling requirements. Therefore, this paper used a multibody dynamics simulation to obtain the rail wear rate.

The main purpose of sampling is to analyze the selected samples and to deduce the characteristics of all the samples. In order to obtain more comprehensive rail wear rate data, this paper takes a sampling method to obtain the samples needed for data fitting. According to the characteristics of rail wear and grinding maintenance, the rail profiles were sampled. As the wear of the rail profile is a continuous change, in order to make the sample profile representation and uniformity and other characteristics, the D-optimal experimental design sample was selected. According to the composition of the curve to be smooth and smooth by the optimization points of rail profile and the geometrical requirements of the rails in the actual railway condition, the D-optimal experimental design was used to sample 101 sets of rail profiles.

Based on the vehicle-track coupling dynamics model and the Archard wear model, the wear of the sample rail profiles was simulated and the wear rate of the rail profiles was obtained.

The railway wagon model adopted a $30 \mathrm{t}$ axle load open wagon. The model assumed that the wheel set, bolsters, side frame, and car body were rigid, regardless of elastic deformation, which was ignored. The mass distribution of bogie and car body was symmetrical. According to related literature, the bogie of a $30 \mathrm{t}$ axle load wagon can be selected according to [22]. The wheel set had six degrees of freedom, respectively, representing longitudinal, lateral, vertical, yaw, roll, and pitch modes. The vehicles were equipped with new wheels (with the Chinese LM wheel profile). The friction coefficient between the wheel and rail was 0.3 .

The track model was assembled on a $600 \mathrm{~m}$ radius curve with $1435 \mathrm{~mm}$ gauge. The track structure included a CHN $75 \mathrm{~kg} / \mathrm{m}$ rail, Type III sleeper, Type II elastic fastener, and ballast bed with a thickness of $0.3 \mathrm{~m}$. The track model was simplified into a massless viscoelastic force with vertical and lateral stiffness and damping. The running railway cars were under irregularity from the track. The Chinese heavy haul railway was similar to the five class track spectrum of the United States [23]. Thus, we selected this spectrum as the random irregularity to conduct simulation.
3.2. SVM Nonlinear Regression. Support vector machine, also known as SVM, is a learning method where computer is used for statistical analysis of data. The rationale of SVM is that the sample relationship identified through summarization and generalization of given sample point data is generalized. The basic means of SVM regression analysis for rail wear rate model is linearization that converts the nonlinear problem between profile and wear rate into the combination of linear problems in higher dimensional space.

In consideration of the rationale of SVM, assuming that $\mathbf{y}=\left(y_{1}, y_{2}, \ldots, y_{12}\right)^{T}$ is input sample vector and $\mathbf{r}$ is the corresponding output value, then

$$
\mathbf{r}=f(\mathbf{y})+v
$$

where $v$ is the offset parameter to be determined.

The nonlinear basis function $\left\{\varphi_{j}(\mathbf{y})\right\}_{j=0}^{n}$ is introduced to express the nonlinearity therebetween, and then (7) could be converted to

$$
\mathbf{r}=\sum_{j=0}^{m} \mathbf{w}_{j} \varphi_{j}(\mathbf{y})=\mathbf{w}^{\mathrm{T}} \boldsymbol{\varphi}(\mathbf{y}) .
$$

Seek the optimal regression surface analysis rationale based on SVM, define two nonnegative slack variables (i.e., $\xi$ and $\eta$ ), constant penalty factor $C$, and insensitive error parameter $\varepsilon$, and realize the conversion into a quadratic convex programming problem through regression of sample data

$$
\begin{array}{ll}
\min & \frac{1}{2}\|\mathbf{w}\|^{2}+C \sum_{i=0}^{m}\left(\xi_{i}+\eta_{i}\right) \\
\text { s.t. } & r_{i}-\left(\mathbf{w} \cdot \mathbf{y}_{i}\right)-b \leq \varepsilon+\xi_{i} \\
& \left(\mathbf{w} \cdot \mathbf{y}_{i}\right)+b-r_{i} \leq \varepsilon+\eta_{i} \\
& (i=1,2, \ldots, m) .
\end{array}
$$

Use the Lagrange multiplier method and introduce operators $\alpha_{i}$ and $\alpha_{i}^{i}$; then the optimal solution to the rail profile optimization problem in higher dimensional space is

$$
\begin{aligned}
f(\mathbf{y}) & =(\mathbf{w} \cdot \mathbf{y})+b \\
& =\sum_{\text {support vector }}\left(\alpha_{i}-\alpha_{i}^{\prime}\right) K\left(\mathbf{y}, \mathbf{y}_{\mathbf{i}}\right)+b,
\end{aligned}
$$

where $K\left(\mathbf{y}, \mathbf{y}_{\mathbf{i}}\right)=\left(\varphi(\mathbf{y}) \cdot \varphi\left(\mathbf{y}_{\mathbf{i}}\right)\right)$.

Where SVM is used for regression of the nonlinear model of rail wear rate and profile, the sample data set is normally divided into two parts, that is, training sample and test sample. Training set is used for SVM training and learning stage, when the computer performs summarization and generalization based on input values and output values in training sample to attain the statistical model of sample data. Test set normally plays no role in the fitting process of 


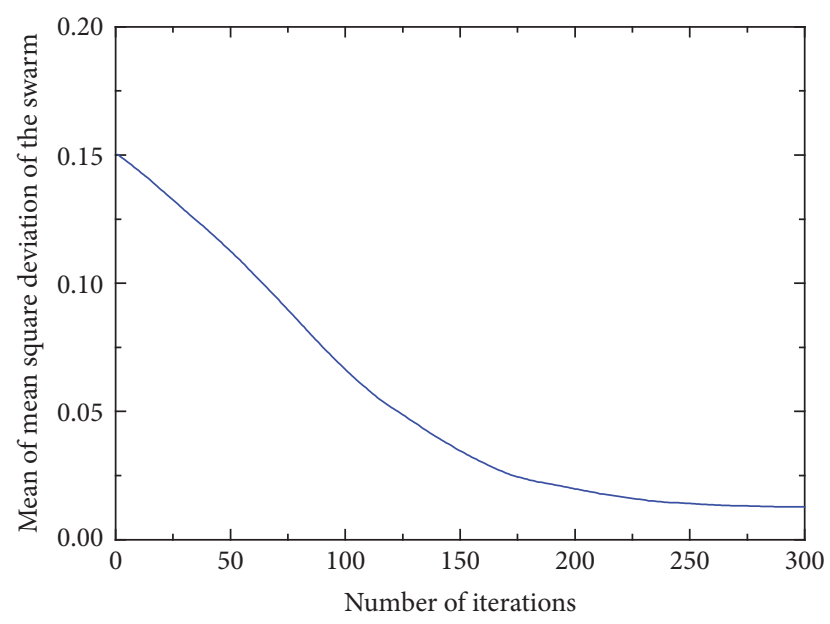

FIGURE 3: Swarm mean curve of particle swarm optimization.

model but is used to verify the prediction accuracy of SVM nonlinear regression upon completion of model training.

3.3. SVM Regression Parameter Optimized with PSO. According to (9) and (10), penalty factor $C$ and kernel function radius $g$ significantly affect the regression prediction accuracy of model during SVM-based model training. Under normal conditions, $C$ is monotonously negatively correlated with fitting error within a certain range [24]. Where the same value is chosen, prediction error nonmonotonically decreases with the increase of penalty factor, but the increase of its value may result in overfitting. Where $g$ value is small, the kernel function curve changes slowly, in which case overfitting may arise during model fitting and affect the prediction; underfitting may arise when $g$ value is small. Hence, particle swarm optimization (PSO for short) is used for optimization and to identify appropriate $C$ and $g$ values during regression of rail profile optimization model.

Mean square deviation is taken as the fitness function of model when SVM parameters $C$ and $g$ are optimized with particle swarm optimization. The obtained relationship between best particle mean square deviation and the number of iterations is shown in Figure 3.

As shown in Figures 3 and 4, swarm mean square deviation gradually decreases with the increase in the number of iterations within a certain range. When the number of particle iterations is less than 200 , the swarm mean square deviation curve changes significantly. When the number of iterations reaches 300 , the training swarm mean square deviation curve gets into a convergence state and converges at 0.013 . The best particle mean square deviation curve substantially tends to be stable after 50 iterations, and the convergence value is 0.012795 . The corresponding optimized kernel function radius $g=0.0068$, and the penalty factor $C$ $=101.8664$.

3.4. Rail Wear Rate Model Analysis. After the optimal kernel function radius $g$ and penalty factor $C$ are obtained through particle swarm optimization, $\mathrm{K}$ cross validation is used to

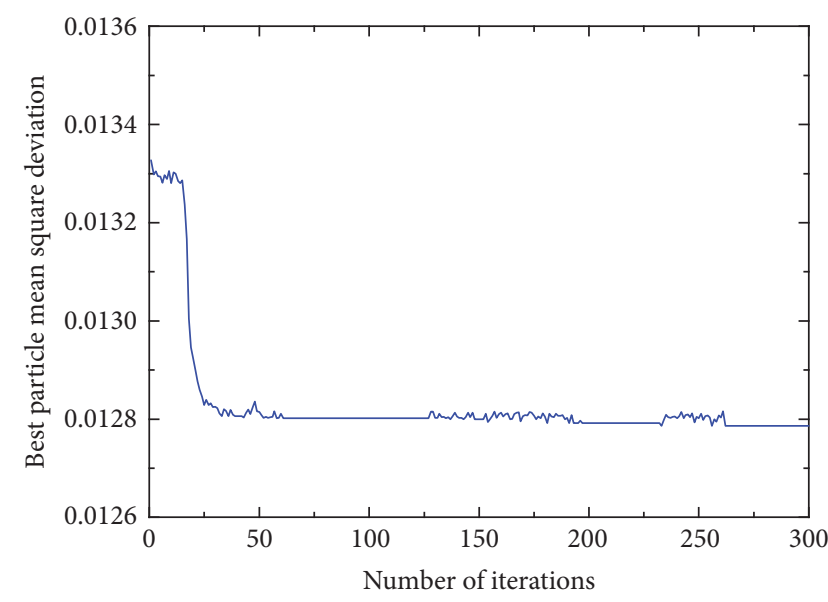

FIGURE 4: Optimal value curve of particle swarm optimization.

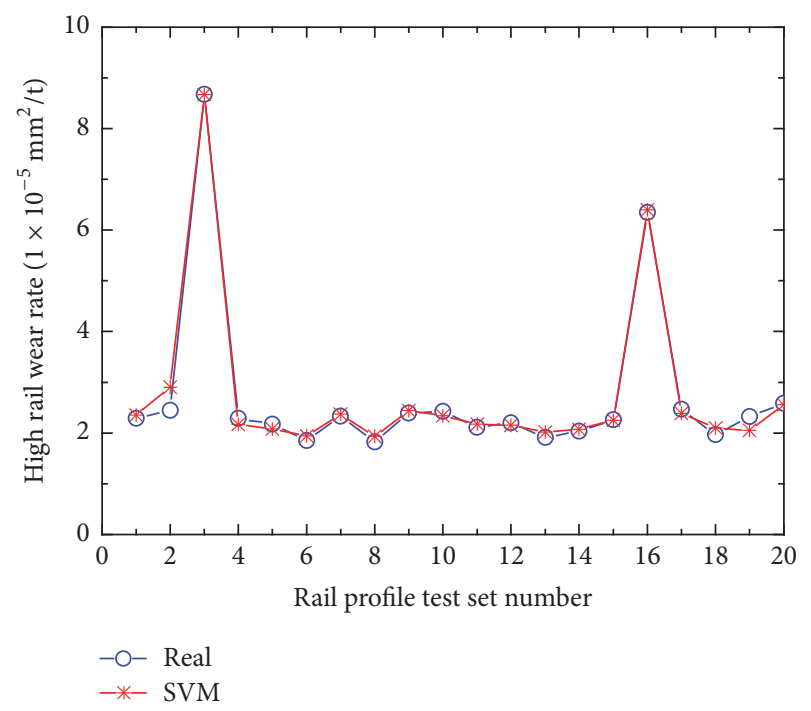

FIGURE 5: Rail profile test set prediction result of wear rate.

subject a certain part of the 101 selected groups of rail profile wear data to random sampling of rail profile. The rail samples taken are divided into training set and test set. Training set is used for modeling during regression, while test set is used to determine the regression accuracy of model. The stability of built model is analyzed and compared through a number of cross combinations. In this paper, 20 groups of data constitute the test set for model; whole others constitute the training set for the regression of rail profile optimization model. Figures 5 and 6 show the prediction result of test sample by PSO-SVM, and Table 2 shows the prediction data of test set by PSO-SVM rail profile optimization model.

As shown in Figures 5 and 6, the predicted value of test sample is extremely close to its actual value, and the variation tendency of predicted value substantially keeps up with that of actual value. According to the prediction data in Table 2, the overall relative error of prediction for test sample is less than 20\%. The prediction errors for Groups 40 and 22 are beyond $10 \%$, while the prediction errors for all other 18 
TABLE 2: Prediction value and simulation value of rail profile test sample (unit: $\mathrm{mm}^{2} / \mathrm{t}$ ).

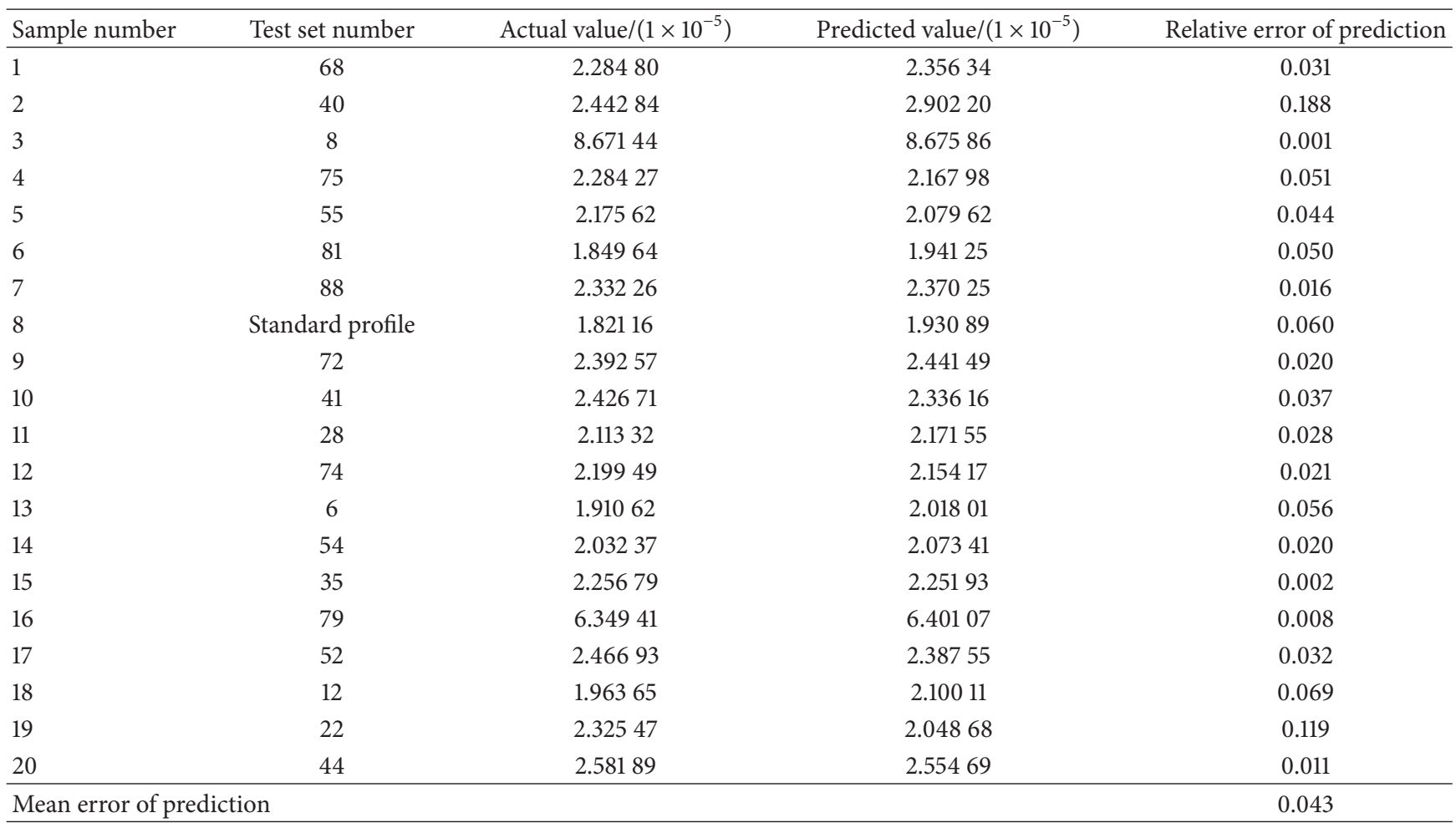

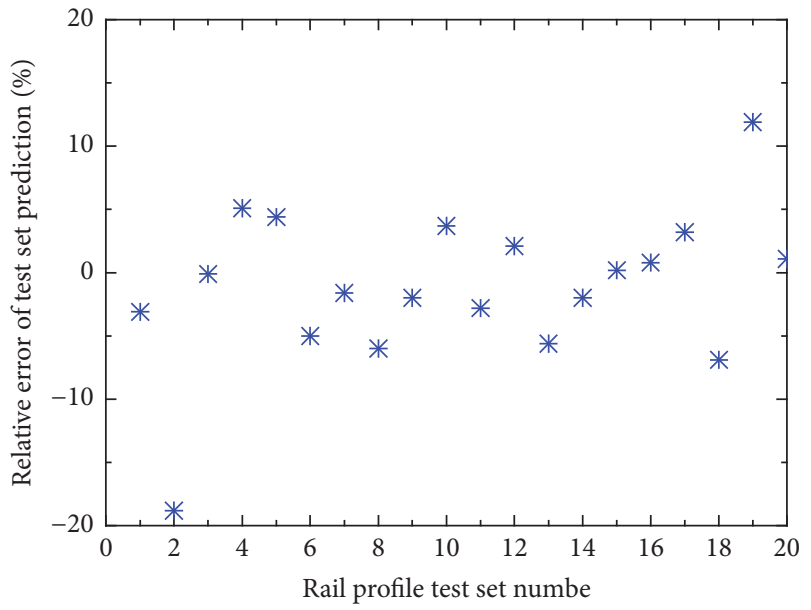

FIGURE 6: Rail test sample prediction wear rate error.

groups of samples are around 5\%. The mean relative error of prediction for the 20 groups of test samples is $4.3 \%$, which means that PSO-SVM regression model for rail wear rate has excellent prediction performance.

\section{Numerical Solution to Rail Profile Optimization Model}

Rail profile optimization involves a number of factors. Rail wear is associated with its material, the acting force between wheel and rail and other wheel-rail system factors, and is closely associated with ambient environment and subsequent maintenance and grinding. Upon the establishment of rail wear rate model, the optimal solution shall be found for rail wear rate model so as to identify the rail profile with minimum wear loss at curved section within design cycle with the purpose of reducing rail wear at curved section and extending the service life of rail as stated in this paper.

With the vertical coordinate of point at rail profile as the independent variable and the wear rate as the dependent variable, this paper identifies the randomness, complexity, multiple constraints, and so forth of nonlinear rail profile optimization model for curved section by subjecting both variables to fitting through PSO-SVM. Genetic algorithm is used to seek numerical solution of this model based on abovenoted features of the model.

4.1. Parameter Setup of Genetic Algorithm. The following issues shall be taken into account when using genetic algorithm to seek to solve rail profile optimization model.

(1) Swarm and Swarm Size. The independent variable $y$ in rail profile optimization model represents gene, so the individual rail profile could be expressed as $\mathbf{y}_{\mathbf{i}}=\left(y_{1 \mathbf{i}}, y_{2 \mathbf{i}}, \ldots, y_{12, \mathbf{i}}\right)^{\mathrm{T}}$, where the aggregate of rail profile $\mathbf{y}_{\mathbf{i}}$ represents the swarm stated in this paper. Generally, the size of swarm is directly proportional to the all-sidedness of genetic algorithm solution, but larger size of swarm may consume longer time of iterative computation. The swarm size is defined as 200 based on requirement of this paper. 


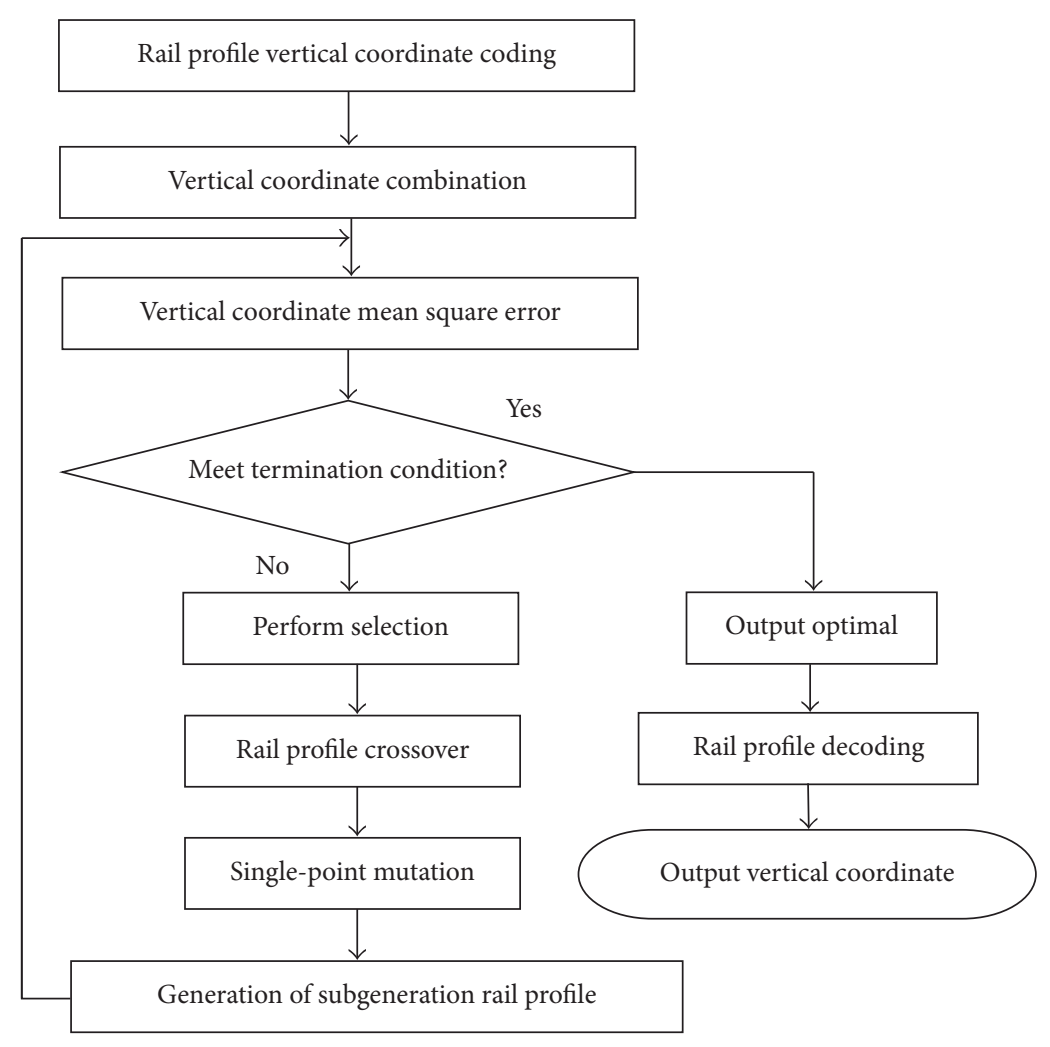

FIGURE 7: Computation flow chart of genetic algorithm.

(2) Selection Operator. According to the "survival of the fittest" principle in genetic algorithm, the operators with more favorable fitness values are more likely to be chosen; otherwise, the individuals will be knocked out. Genetic operators in common use include proportional selection, the best individual preservation strategy, and deterministic sampling [25], and this paper takes proportional selection as selection operator.

(3) Crossover Operator $p_{c}$. Where genetic algorithm is concerned, "crossover" means genetic recombination; that is to say, two individuals exchange gene with each other in a certain way to generate a new individual. This paper performs two-point crossover for chromosome in the following procedure.

Assume that $\mathbf{y}_{1}=\left(y_{1}^{1}, y_{2}^{1}, y_{3}^{1}, \ldots, y_{11}^{1}, y_{12}^{1}\right)^{\mathrm{T}}$ and $\mathbf{y}_{2}=$ $\left(y_{1}^{2}, y_{2}^{2}, y_{3}^{2}, \ldots, y_{11}^{2}, y_{12}^{2}\right)^{\mathrm{T}}$; if gene exchanges locations $r_{1}=3$ and $r_{2}=11$, the chromosome after two-point crossover should be $\mathbf{y}_{1}^{\prime}=\left(y_{1}^{1}, y_{2}^{1}, y_{3}^{2}, \ldots, y_{11}^{2}, y_{12}^{1}\right)^{\mathrm{T}}$ and $\mathbf{y}_{2}^{\prime}=\left(y_{1}^{2}, y_{2}^{2}\right.$, $\left.y_{3}^{1}, \ldots, y_{11}^{1}, y_{12}^{2}\right)^{\mathrm{T}}$.

(4) Mutation Operator $p_{m}$. Mutation offers the genes omitted during computation, enables the global search of rail profile, and helps to avoid precocity. Single-point mutation is employed in rail wear profile optimization model. The operating procedure is as follows.
For chromosome $\mathbf{y}=(1.1,1.5,2.2,2.3, \ldots, 13)^{\mathrm{T}}$, the mutation position is 2 , and then $\mathbf{y}^{\prime}=(1.1,1.8,2.2,2.3, \ldots, 13)^{\mathrm{T}}$ is obtained after random mutation.

The process of seeking optimal rail profile through genetic algorithm is shown in Figure 7.

4.2. Rail Profile Optimization Result. Figure 8 shows the optimized profile Opt that meets constraint condition and is computed by seeking to solve PSO-SVM rail profile optimization model through genetic algorithm.

Figure 9 shows the variation of vertical coordinates of the optimized profile and standard profile. Thus it can be seen that the vertical coordinate difference at optimization point increases with the increase of horizontal coordinate. When the horizontal coordinate of optimization point is $(-10,20)$, the vertical coordinate changes slowly (normally no more than $0.5 \mathrm{~mm}$ ) at points in front and behind this point; when the optimization point is $(20,36)$, its vertical coordinate changes significantly, and the vertical coordinate difference increases with the increase of horizontal coordinate before and after optimization. The rail profile changes significantly when horizontal coordinate is located between $20 \mathrm{~mm}$ and $36 \mathrm{~mm}$, and the maximum depth of Opt and standard profile optimization point variation is $1.9004 \mathrm{~mm}$. This is principally because the overall linearity of rail head exhibits a convex curve. Since the most frequent rail wear at curved section is 


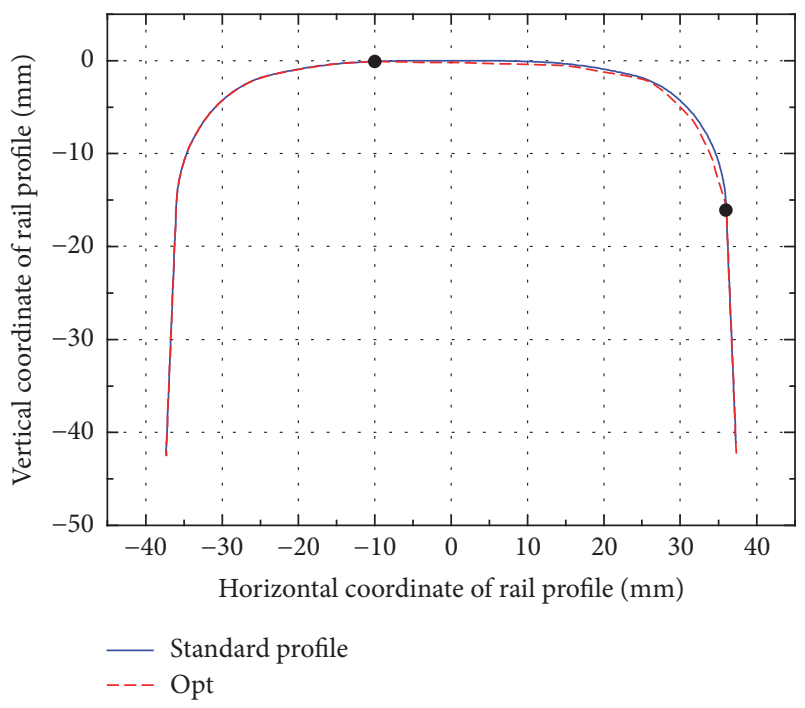

Figure 8: Comparison between optimized profile and standard profile.

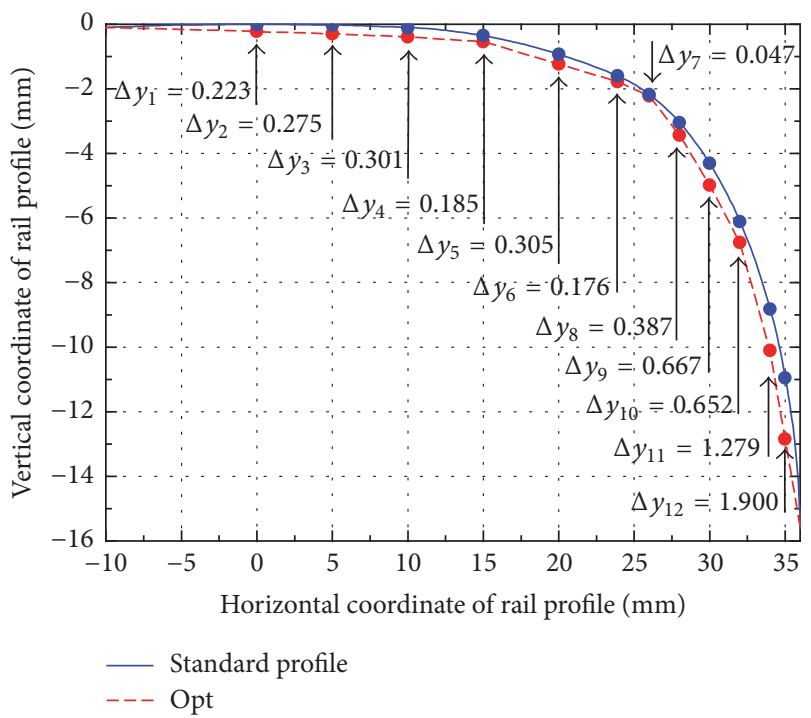

FIGURE 9: Local comparison of optimization interval (unit: mm).

side wear, the optimization depth at rail side shall be greater than at top face in order to reduce the contact between rail side and wheel flange and concentrate the wheel-rail contact at top of rail head to the greatest extent so as to minimize rail wear.

\section{Analysis of Performance of Optimized Rail Profile}

5.1. Wear Analysis. Genetic algorithm solution seeking and simulation calculation show that the optimized rail profile offers lower wear rate and improves the allowable gross rail load on axle as compared with standard profile under the same conditions within an established wear cycle, having reached the research objective of this paper.

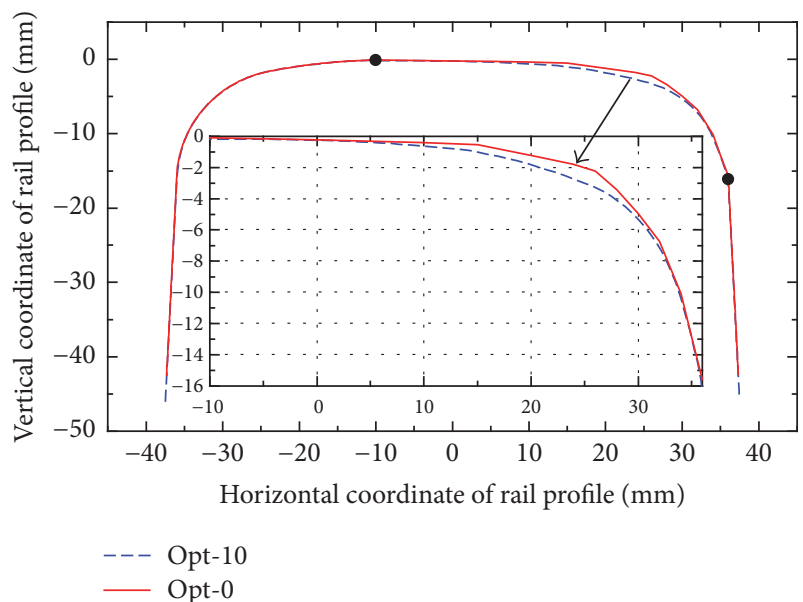

Figure 10: Profiles before and after wear of the optimized profile.

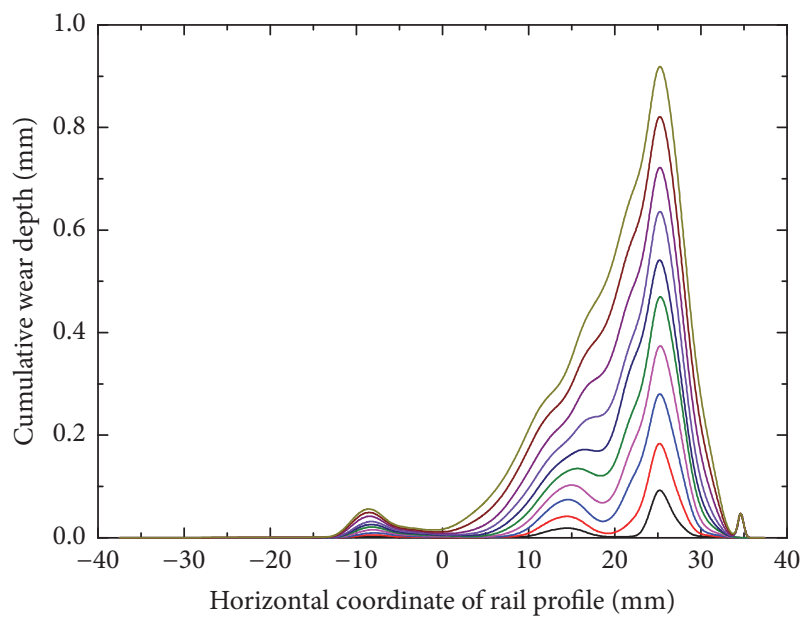

FIGURE 11: Accumulation of the optimized profile wear.

In view of the progressive accumulation of rail wear, $1 \mathrm{~mm}$ of the profile wear depth was taken as rail wear analysis cycle; that is to say, the termination condition for simulation of rail profile wear was that the maximum wear depth reached $1 \mathrm{~mm}$. In this range, the variation of the optimized profile wear determined through rail wear model simulation calculation, shown in Figure 10. Opt-0 is the optimized rail profile, and Opt-10 is the rail profile when wear depth reaches $1 \mathrm{~mm}$. Figure 10 shows the accumulative variation of rail optimized profile wear depth.

It is observed from Figures 10 and 11 that most wear of high rail before and after optimization is located at horizontal coordinate $10-30 \mathrm{~mm}$. Wear simulation calculation showed that the allowable number of passes at the optimized profile Opt is 707,605 vehicles, while the allowable gross load is $84.913 \mathrm{Mt}, 12.7 \%$ up as compared with standard profile. The wear rate simulation value of the optimized profile Opt is $1.71562 \times 10^{-5} \mathrm{~mm}^{2} / \mathrm{t}, 5.8 \%$ down as compared with standard profile.

The wear rate of the optimized profile Opt predicted by genetic algorithm (PSO-SVM Regression) is $1.61702 \times$ $10^{-5} \mathrm{~mm}^{2} / \mathrm{t}$. Simulations comparison analysis showed that 
TABLE 3: Maximum values of dynamics index of train in the case of optimized profile.

\begin{tabular}{lcccccccrrr}
\hline \multirow{2}{*}{ Safety index } & \multicolumn{2}{c}{ Wheelset 1 } & \multicolumn{2}{c}{ Wheelset 2 } & \multicolumn{2}{c}{ Wheelset 3 } & \multicolumn{2}{c}{ Wheelset 4 } & \multirow{2}{*}{ Standard values } \\
& 1L & 1R & 2L & 2R & 3L & 3R & 4L & 4R & 0.8 \\
Derailment coefficient & 0.5246 & 0.5196 & 0.4015 & 0.4033 & 0.5648 & 0.3750 & 0.3372 & 0.4720 \\
Wheel-rail lateral force/kN & 93.169 & 59.631 & 60.741 & 68.655 & 70.991 & 41.896 & 42.635 & 52.804 \\
Wheel load reduction rate & \multicolumn{2}{c}{0.637} & \multicolumn{2}{c}{0.541} & \multicolumn{2}{c}{0.489} & & 0.58 & 180 \\
\hline
\end{tabular}

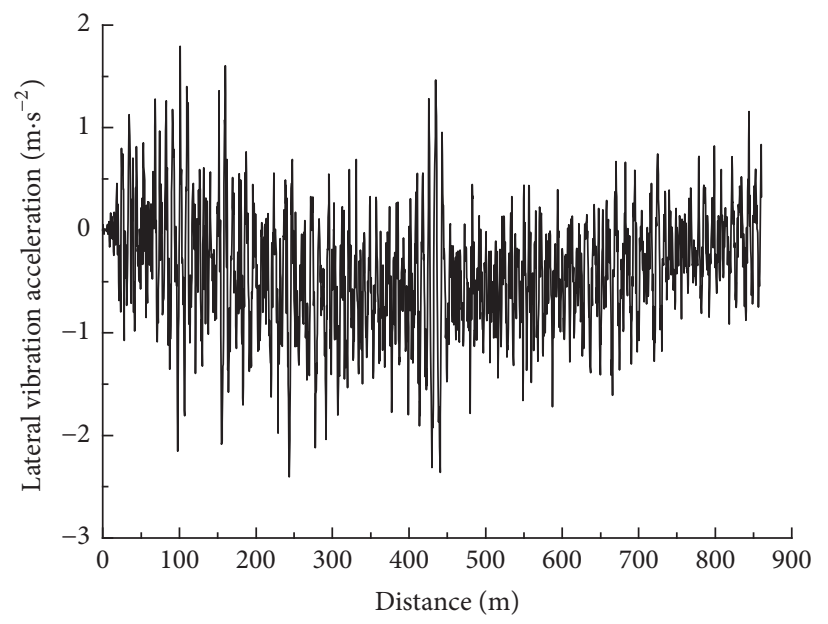

(a) Lateral acceleration curve

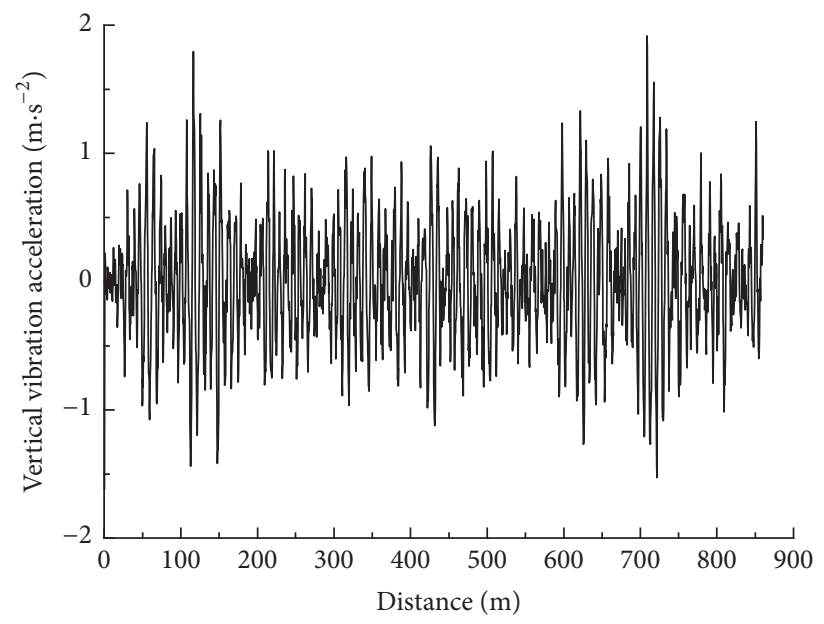

(b) Vertical acceleration curve

FIGURE 12: Vibration acceleration curve in the case of optimized profile.

the relative error between rail wear prediction value and simulation value is $6 \%$. To sum up, the result of optimization of rail profile with mathematical model of rail wear presented in this paper is consistent with optimization objective.

5.2. Dynamic Performance Check for Optimized Profile. Carriage safety and stability constitute the prior condition for all maintenance and repair of rails. Dynamics analysis was performed on the motion state of a freight train with $30 \mathrm{t}$ axle load passing through a curved section of $600 \mathrm{~m}$ in radius at $72 \mathrm{~km} / \mathrm{h}$. The safety and stability indicators like dynamic derailment coefficient, wheel-rail lateral force, wheel load reduction rate, and vibration acceleration of a train passing through a curved section were calculated with optimized profile as object of study under the same simulation conditions. Table 3 shows the maximum values of safety index for each wheelset in the case of optimized profile.

The body vibration acceleration curve of a train along the established route in the case of optimized profile is shown in Figure 12.

The calculation values in Table 3 show that, according to the provisions of Dynamic Performance Test Appraisal Method and Evaluation Criteria for Rolling Stock (TB/T23601993) on above-noted indicators [26, 27], the train complies with safety index during operation on optimized profile. According to the provisions of GB 5599-85 on vibration acceleration of freight trains that the vertical vibration acceleration shall not be greater than $0.7 \mathrm{~g}\left(6.86 \mathrm{~m} / \mathrm{s}^{2}, g\right.$ is the gravitational acceleration) while the lateral acceleration shall not be greater than $0.5 g\left(4.90 \mathrm{~m} / \mathrm{s}^{2}\right)$, Figure 12 shows that the stability index of train traveling on optimized profile is satisfactory.

\section{Conclusion}

This paper established a mathematical model for rail profile optimization with purpose of reducing rail wear rate. During the mathematical modeling, the wear characteristics of curved portion of rail were taken into consideration while the 12 discrete points selected in optimization interval were used for spline interpolation to indirectly express the geometric profile of rail. To simplify the modeling process, unit-length rail was taken as the subject investigated, while the rail wear volume was translated into wear area, which could be calculated through the difference between profiles before and after wear. In such a manner, the independent variable and dependent variable of mathematical model were, respectively, numeralized. The following conclusion was reached:

(1) Support vector machine was used for regression fitting of rail wear model based on sample rail profile simulation data. To improve the generalization capability of regression model and reduce the model prediction error, particle swarm optimization was proposed to optimize the kernel function radius $g$ and penalty coefficient $C$ in support vector machine regression. Rail wear rate model was built after sample training with PSO-SVM. The prediction of test set identified that the average relative error of test samples in this paper was $4.3 \%$. After a number of training 
processes on the model, the average relative error of test set was kept below $10 \%$.

(2) Genetic algorithm was used to solve rail profile optimal model to get the optimal rail profile based on optimality principle. According to simulation calculation, the wear rate of optimized profile Opt decreased by $5.8 \%$, while the allowable gross load increased by $12.7 \%$ as compared with standard profile.

(3) The analysis of train operation safety and stability on the optimized rail profile Opt performed with derailment coefficient, wheel-rail lateral force, wheel load reduction rate, and vibration acceleration as indicators showed that the optimized rail profile Opt met normal travel conditions of train.

In consideration of all characteristics mentioned above, this mathematical model is available to optimize the design of curved portion profile of rail.

This paper takes into account the profile variation during service of rail and leaves out of consideration the diversity of wheel profile and the wear-induced change in wheel profile, which constitute the shortcoming of this study. To make up for such shortcoming, it is advisable to carry out in-depth research using the techniques similar to that stated in this paper, so as to obtain optimized rail profile with wheel-rail wear within the interval taken into account, thereby getting closer to practical situation.

\section{Conflicts of Interest}

The authors declare that there are no conflicts of interest regarding the publication of this paper.

\section{Acknowledgments}

This work was supported by National Natural Science Foundation of China (no. 51208318 and no. 51208319), Hebei Provincial Natural Science Foundation of China (no. E2015210099 and no. E2016210131), and Key Project of Hebei Educational Committee of China (no. ZD2015037 and no. ZD2014084).

\section{References}

[1] X. Chen, Research on the Influence of Curve Parameter on Rail Wear for Heavy Haul Railway, Shi jia zhuang Tie Dao University, Shi jia zhuang, China, 2015.

[2] E. E. Magel and J. Kalousek, "The application of contact mechanics to rail profile design and rail grinding," Wear, vol. 253, no. 1-2, pp. 308-316, 2002.

[3] I. Y. Shevtsov, Wheel/Rail Interface Optimization, Delft University of Technology, Delft, Netherlands, 2008.

[4] J. Jia and D. Si, "Target profile of rail grinding for small radius curve of Shuohuang Railway," China Railway Science, vol. 35, no. 4, pp. 15-21, 2014.

[5] W. Zhai, J. Gao, P. Liu, and K. Wang, "Reducing rail side wear on heavy-haul railway curves based on wheel-rail dynamic interaction," Vehicle System Dynamics, vol. 52, no. 1, pp. 440454, 2014
[6] J. Brandau, G. Voß, and G. Poll, "Wear reduction in light rail systems through asymmetrical railhead profiles," Tribology Series, vol. 40, pp. 305-316, 2002.

[7] H. Y. Choi, D. H. Lee, C. Y. Song, and J. Lee, "Optimization of rail profile to reduce wear on curved track," International Journal of Precision Engineering and Manufacturing, vol. 14, no. 4, pp. 619625, 2013.

[8] J. Xiao, The Theory and Test Research on Rail Asymmetric Grinding for the Passenger and Freight Railway, Southwest Jiaotong University, Chengdu, China, 2011.

[9] R. P. B. J. Dollevoet, Design of An Anti Head Check Profile Based on Stress Relief, Enschede, Netherlands, University of Twente, 2010.

[10] D. B. Cui, L. Li, X. S. Jin, and L.-J. Zhou, "Study on rail goal profile by grinding," Engineering Mechanics, vol. 28, no. 4, pp. 178-184, 2011.

[11] I. Persson, R. Nilsson, U. Bik, M. Lundgren, and S. Iwnicki, "Use of a genetic algorithm to improve the rail profile on Stockholm underground," Vehicle System Dynamics, vol. 48, supplement 1, pp. 89-104, 2010.

[12] B. A. Pålsson, "Design optimisation of switch rails in railway turnouts," Vehicle System Dynamics, vol. 51, no. 10, pp. 1619-1639, 2013.

[13] G. Shen and X. Zhong, "Implementations of newly developed wheel and rail profile design methods," Journal of Traffic and Transportation Engineering, vol. 1, no. 3, pp. 221-227, 2014.

[14] H. Jahed, B. Farshi, M. A. Eshraghi, and A. Nasr, "A numerical optimization technique for design of wheel profiles," Wear, vol. 264, no. 1-2, pp. 1-10, 2008.

[15] J. Gerlici and T. Lack, "Railway wheel and rail head profiles development based on the geometric characteristics shapes," Wear, vol. 271, no. 1-2, pp. 246-258, 2011.

[16] P. Wang, G. Liang, X. Tao et al., "Study on the numerical optimization of rail profiles for heavy haul railways," in Journal of Rail and Rapid Transit, 2016.

[17] L. Zhou, J. Liu, D. Cui, and et al., "Profile study of preventive rail grinding in high-speed railway based on trains running performance," Railway Locomotive \& Car, vol. 30, no. 5, pp. 3439, 2010.

[18] S. Zakharov, I. Goryacheva, V. Bogdanov et al., "Problems with wheel and rail profiles selection and optimization," Wear, vol. 265, no. 9-10, pp. 1266-1272, 2008.

[19] G. V. Bergeman, "Development of a new progressive rolling technology of profile of neckless grooved tram rail," Metallurgical and Mining Industry, vol. 7, no. 1, pp. 95-101, 2015.

[20] X. Meng, "Experimental study on wear-reducing measures of small radius curve rail in heavy haul railway," China Railway, vol. 5, pp. 37-41, 2013.

[21] Z. Chen, Optimization Design and Improvement Analysis of Rail Profile Based on Vehicle Dynamics for Curve Wear at Heavy Haul Railway, Southwest Jiaotong University, Chengdu, China, 2009.

[22] F. Qi, Research on Key Technology of 30t Axle-Load Bogie [Master's, thesis], Southwest Jiaotong University, PRC, 2009.

[23] Y. Geng and Y. Hu, "On simulating track spectrum of rail vehicle dynamics," Research on City Orbit Traffic, vol. 7, pp. 20-23, 2010.

[24] J. Tian and M. Gao, Artificial Neural Network Algorithm Research and Application, Beijing Institute of Technology Press, Beijing, China, 2006.

[25] Q. Xiao, "Realization of several commonly used selection operators in genetic algorithm," Computer and Digital Engineering, vol. 33, no. 9, pp. 140-142, 2005. 
[26] Z. Ren, Vehicle System Dynamics, China Railway Publishing House, Beijing, China, 2007.

[27] Ministry of Railway of the People's Republic of China, Testing method and evaluation standard for dynamic performance of railway locomotives (TB/T2360-1993), China Railway Publishing House, Beijing, China, 1993. 


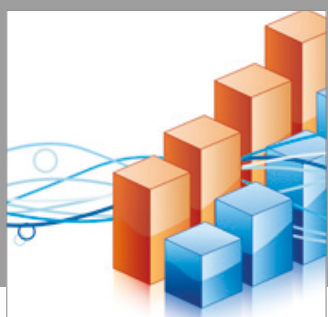

Advances in

Operations Research

vatersals

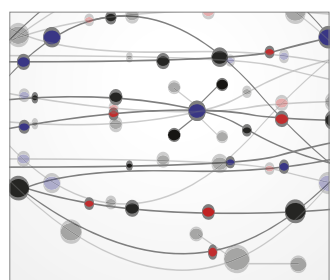

\section{The Scientific} World Journal
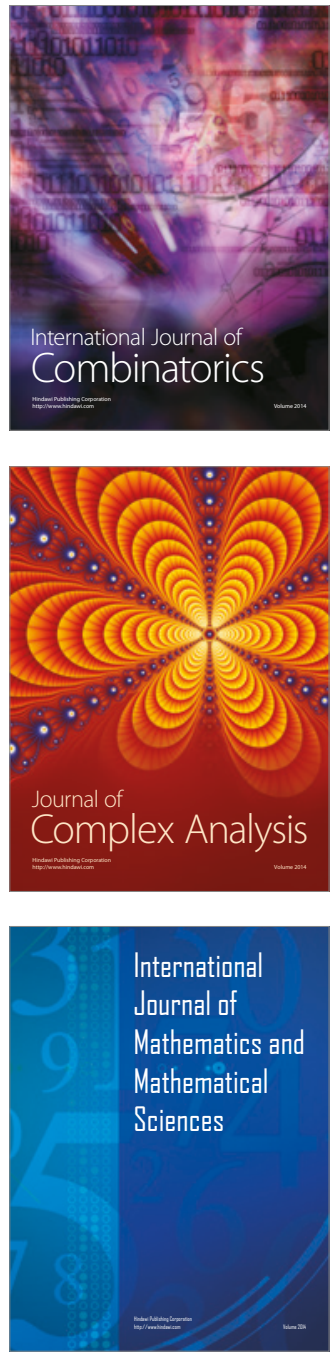
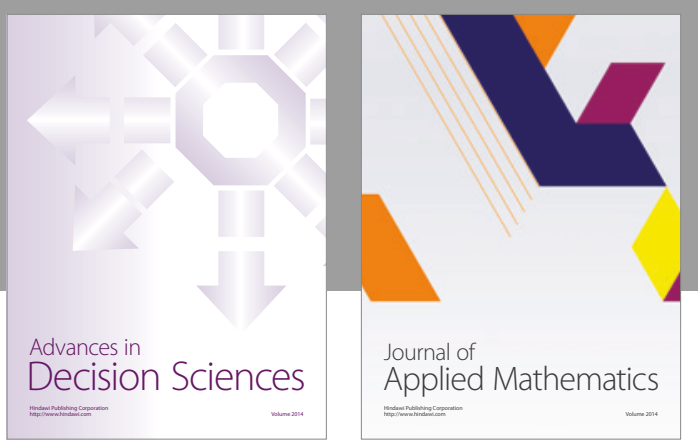

Algebra

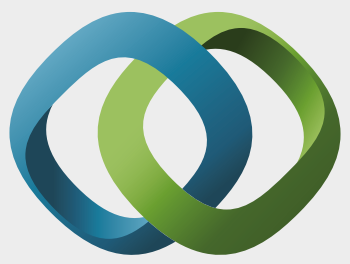

\section{Hindawi}

Submit your manuscripts at

https://www.hindawi.com
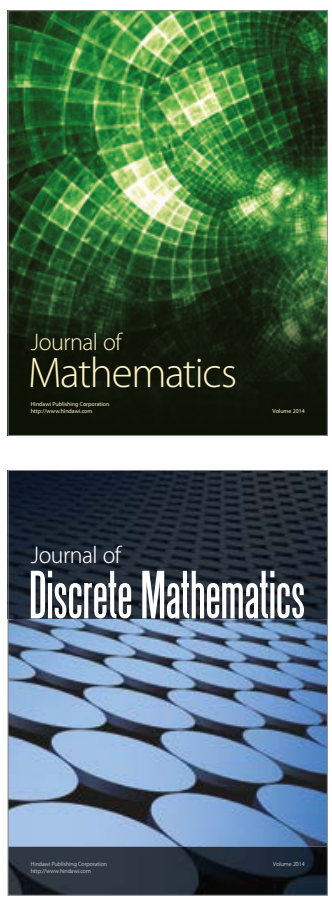

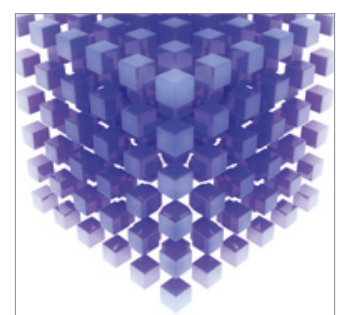

Mathematical Problems in Engineering
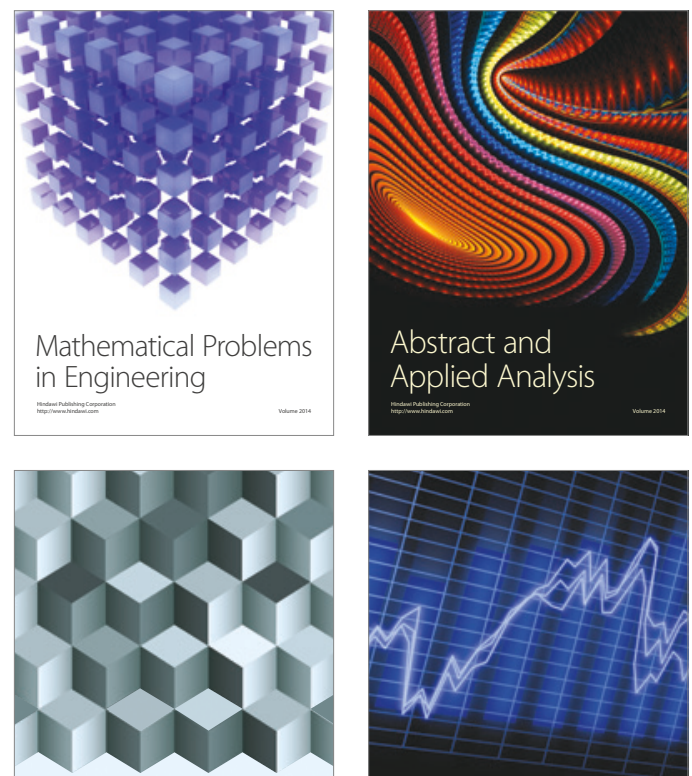

Journal of

Function Spaces

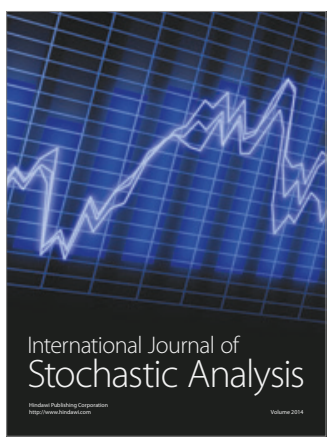

Probability and Statistics
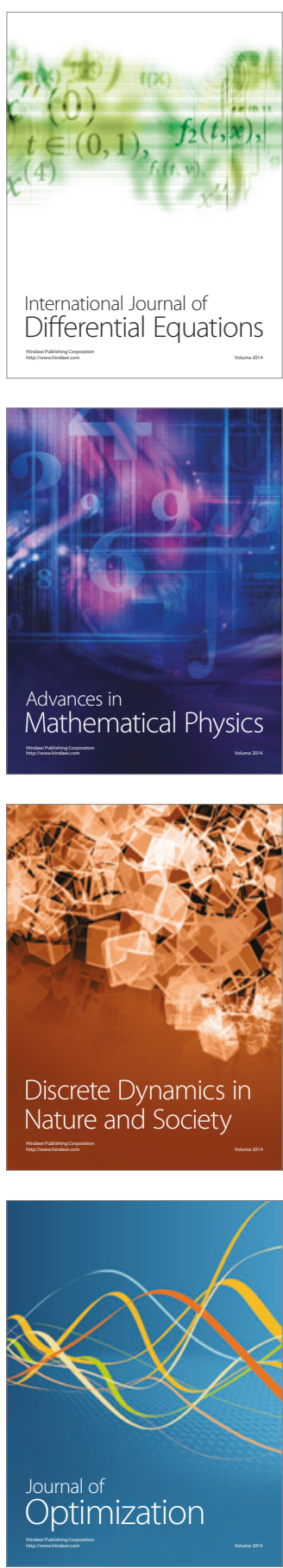